\title{
Experimental and numerical analysis of flow instabilities in rectangular shallow basins
}

\author{
B.J. Dewals · S.A. Kantoush • S. Erpicum • \\ M. Pirotton · A.J. Schleiss
}

Received: 22 June 2007 / Accepted: 7 January 2008 / Published online: 6 February 2008

(C) Springer Science+Business Media B.V. 2008

\begin{abstract}
Free surface flows in several shallow rectangular basins have been analyzed experimentally, numerically and theoretically. Different geometries, characterized by different widths and lengths, are considered as well as different hydraulic conditions. First, the results of a series of experimental tests are briefly depicted. They reveal that, under clearly identified hydraulic and geometrical conditions, the flow pattern is found to become nonsymmetric, in spite of the symmetrical inflow conditions, outflow conditions and geometry of the basin. This non-symmetric motion results from the growth of small disturbances actually present in the experimental initial and boundary conditions. Second, numerical simulations are conducted based on a depth-averaged approach and a finite volume scheme. The simulation results reproduce the global pattern of the flow observed experimentally and succeed in predicting the stability or instability of a symmetric flow pattern for all tested configurations. Finally, an analytical study provides mathematical insights into the conditions under which the symmetric flow pattern becomes unstable and clarifies the governing physical processes.
\end{abstract}

Keywords Depth-averaged model · Finite volume · Reservoir hydrodynamics · Shallow flow $\cdot$ Stability analysis

\section{Introduction}

Flows in wide and shallow open channels may become unstable, leading to large-scale transverse motion and eddies, as a result of the growth of transverse disturbances, due to the

\footnotetext{
B.J. Dewals $(\varangle) \cdot$ S. Erpicum $\cdot$ M. Pirotton

Laboratory of Applied Hydrodynamics and Hydraulic Constructions, Department ArGEnCo - Secteur $\mathrm{MS}^{2} \mathrm{~F}, \mathrm{HACH}$ - Hydrodynamique Appliquée et Constructions Hydrauliques, University of Liege (ULG), Chemin des Chevreuils, 1-Bât B52/3, 4000 Liege, Belgium

e-mail: B.Dewals@ulg.ac.be
}

S.A. Kantoush · A.J. Schleiss

Laboratory of Hydraulic Constructions, Ecole Polytechnique Fédérale de Lausanne (EPFL), Station 18, 1015 Lausanne, Switzerland

B.J. Dewals

Belgian National Fund for Scientific Research (F.R.S.-FNRS), Brussels, Belgium 
high sensitivity of the flow to initial and boundary conditions. There is a keen interest in understanding such flows because of their prominence in nature and their practical importance in many open channel applications, such as jets and wakes [1], flows in compound channels [2,3], in sudden enlargements [4], in sewage manholes or chambers [5,6], as well as in shallow reservoirs [7-9]. Moreover, these large-scale motions influence processes such as sediment and pollutant transport and thus are likely to affect water quality.

After summarizing the results of experimental work carried out at the Laboratory of Hydraulic Constructions (LCH) of the Ecole Polytechnique Fédérale de Lausanne (EPFL), the present paper covers the numerical modelling and the theoretical analysis of the flow in a rectangular shallow basin with varying width $(0.5-4 \mathrm{~m})$ and varying length $(3-6 \mathrm{~m})$. The detailed analysis of those rectangular basins serves as a reference case in the framework of a research project dealing with the sedimentation of shallow reservoirs. In this context, the present study contributes to a better understanding of the influence of the geometry of the reservoir on the flow field and consequently on sediment deposition.

Kantoush (2007) provides a comprehensive review of experimental observations of shallow flows with transverse motion [7], with a focus on flows through symmetric channel expansions. All these observations confirm that even if experimental setups are geometrically and hydraulically symmetric, asymmetric flow patterns can develop under certain geometric and hydraulic conditions, as shown for instance by Stovin [5,6]. Kolyshkin and Ghidaoui (2003) summarize similar findings for wake flows [10], including notably the detailed analysis of shallow flows behind various obstacles carried out by Chen and Jirka [11] Mizushima and Shiotani $(1996,2001)$ [12,13] have studied experimentally and numerically flows in symmetric channels with a suddenly expanded and contracted part for Reynolds numbers lower than 1,500 (in the approaching channel). The present study investigates flows with Reynolds numbers one to two orders of magnitude higher. Consistently with the aforementioned authors, the Reynolds number is defined here as $R e=u b /(2 v)$ ( $u$ and $b$ represent, respectively, the velocity in the inlet channel and the width of the inlet channel, while $v$ is the kinematic viscosity).

Among other numerical studies of flows in open channels with discontinuous expansion and contraction, Mizushima and Shiotani (1996, 2001) [12,13] have used three different methods: time-marching centred finite differences, Successive Over Relaxation (SOR) iterative method and finite elements. A finite volume procedure was used by Battaglia et al. (1997) [14] for channels with sudden expansion only. Commercial CFD codes were employed by several authors, such as Adamsson et al. (2003) [5], for the study of sedimentation in storage tanks, or Kantoush et al. (2005) [15] for the analysis of shallow reservoirs. In the present work, an upwind finite volume scheme, developed at the University of Liege [16,17], is used in combination with a depth-average $k-\varepsilon$ turbulence model [17]. The numerical results are discussed notably in terms of an original quantifier of asymmetry introduced by the authors.

Shapira et al. (1990) [4] applied a linear stability analysis to the flow in a gradual expansion symmetric about its centreline $(50<R e<300)$. They showed the existence of steady non-symmetrical solutions. A finite element algorithm was used to solve for the evolution of disturbances. Chu et al. (1991) [3] used a linear analysis to verify the stability of idealized transverse velocity profiles in compound open channels, based on the rigid-lid approximation and a Runge-Kutta routine combined with a shooting procedure. Beyond discussing the effect of bed friction, they confirmed the important role of the inflection point in the velocity profile [18,19]. Later on, Chen and Jirka (1997) [1] analyzed plane wakes under the rigid-lid approximation. They derived a modified Orr-Sommerfeld equation for open channel flow with friction. They also distinguish absolute and convective instabilities by allowing the wave number to be complex. Ghidaoui and Kolyshkin (1999) [2] showed that when the Reynolds 
number is larger than 1,000 its influence on the flow stability becomes very weak. They also revealed that the rigid-lid assumption is valid for low Froude numbers $(F r=u / \sqrt{g h}$, with $u$ the flow velocity, $g$ the gravity acceleration and $h$ the water depth), as in the present study $(F r<0.1)$. This assessment of the accuracy of the rigid-lid approximation has been confirmed by Kolyshkin and Ghidaoui (2003) [10]. Finally, Mizushima and Shiotani (1996, 2001) $[12,13]$ applied linear stability analysis to flows in channels with sudden expansion and contraction for moderate Reynolds number ( $R e \sim 10^{3}$ or lower). In particular, they investigated the effect of the aspect ratio of the basin (see Sect.2) on the stability of the flow. In the present paper, a linear stability analysis is applied.

In the following sections, the experimental, numerical and theoretical parts of the present research are successively detailed. Next section briefly describes the findings of the experimental tests. The depth-averaged numerical model, as will be exploited for all simulations, is depicted in Sect. 3, while Sect. 4 highlights the ability of the model to represent the non-symmetric motion in the basin. In Sects. 5 and 6, numerical results are compared with experimental measurements for eight different geometries and four different hydraulic conditions, showing a satisfactory agreement between predicted and observed global flow patterns. Finally, a linear sensitivity analysis is carried out in Sect. 7, while Sect. 8 summarizes the results and includes some concluding remarks.

\section{Physical modelling}

The experimental tests have been conducted in a rectangular shallow basin with inner maximum dimensions of $6 \mathrm{~m}$ in length and $4 \mathrm{~m}$ in width, as sketched in Fig. 1. The inlet and outlet rectangular channels are both $0.25 \mathrm{~m}$ wide and $1.0 \mathrm{~m}$ long. The bottom of the basin is flat and consists in hydraulically smooth PVC plates. The walls, also in PVC, can be moved to modify the geometry of the basin. The main measurement techniques employed include ultrasonic probes for measuring water levels, an Ultrasonic Velocity Profiler device (UVP) for measuring 3D velocity components as well as a Large Scale Particle Image Velocimetry technique (LSPIV) for measuring surface velocity fields. A more detailed description of the experimental setup and measurement equipment is given in Kantoush et al. (2005, 2006) $[8,15]$.

The flow has been observed and analysed for eight different basin geometries. These geometries differ by the value of the basin width $B$ and length $L$, as summarized in Table 1 . In all cases, the discharge is kept constant at $Q=7.01 / \mathrm{s}$ and the water level is controlled by a flap gate located in the downstream part of the outlet channel. Except for the tests described in Sect. 6, the downstream water level is kept constant at $h_{0}=0.2 \mathrm{~m}$. Therefore, in all tested configurations, the Reynolds and Froude numbers evaluated in the inlet channel are constant: $R e=1.75 \times 10^{4} ; \mathrm{Fr}=0.10$, except in Sect. 6, where the Froude and Reynolds number are given explicitly (Table 3 ).

Two non-dimensional geometrical parameters are defined for the further analysis (see Sect. 5): the lateral expansion ratio (ER), characterizing the sudden enlargement at the transition between the inlet channel and the basin: $E R=B / b$ and the aspect ratio $(A R)$, characterizing the geometry of the basin: $A R=L / B$. The first parameter holds for describing the influence of a varying width of the basin, while the second one is suitable for describing the effect of variations in the length of the basin. It must be outlined that the width $b$ of the inlet channel, which is used to define the dimensionless parameter $E R$, is kept constant throughout the present study. Therefore, the scalability of the hereafter obtained results with respect to this parameter should be verified based on a separate set of experimental data. 


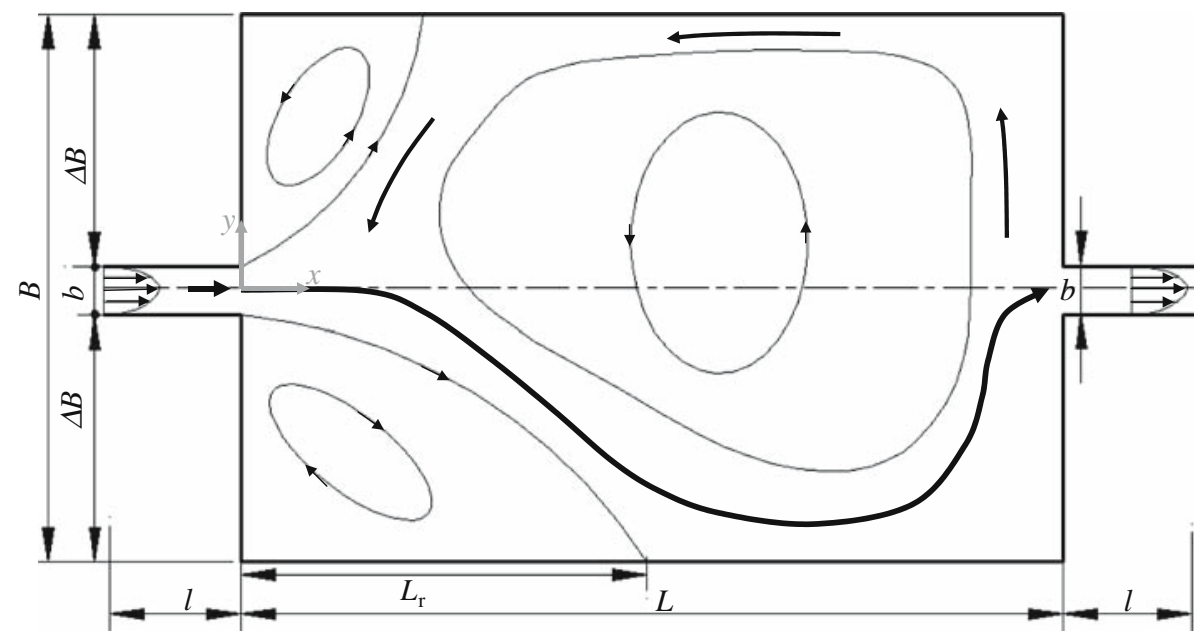

Fig. 1 Plan view of the experimental rectangular basin, definition of the geometrical parameters and sketch of the flow pattern in test no. 1 (see Table 1)

Table 1 Configurations of tested geometries and corresponding geometrical parameters

\begin{tabular}{lllll}
\hline Test no. & $L(\mathrm{~m})$ & $B(\mathrm{~m})$ & $L / B(-)$ & $B / b(-)$ \\
\hline 1 & 6.0 & 4.0 & 1.5 & 16 \\
2 & 5.0 & 4.0 & 1.25 & 16 \\
3 & 4.0 & 4.0 & 1.0 & 16 \\
4 & 3.0 & 4.0 & 0.75 & 16 \\
5 & 6.0 & 3.0 & 2 & 12 \\
6 & 6.0 & 2.0 & 3 & 8 \\
7 & 6.0 & 1.0 & 6 & 4 \\
8 & 6.0 & 0.5 & 12 & 2 \\
\hline
\end{tabular}

In spite of the symmetric setup, an asymmetric flow pattern is observed experimentally for tests no. 1, 5, 6 and 7. On the contrary, the flow remains mainly symmetric for tests no. 2, 3, 4, and 8. Figure 2 shows observed flow fields and streamlines for tests no. 1 and no. 4 $[8,15]$.

It can be noticed (Fig. 2a) that the jet issuing from the inlet channel is considerably deviated in test no. 1 and three large scale vortices develop, including a main large one rotating anticlockwise in the centre part of the basin. Furthermore, two smaller vortices rotating clockwise are formed in the upstream corners of the basin.

The system tends to deviate alternatively to one side or to the other one, depending on slight disturbances existing in the initial and boundary conditions. This deviation is thus observed to be random, indicating that there is no systematic effect, as discussed by Kantoush (2007) [7] and Kantoush et al. (2006) [8], who state that a stable mirror image of the flow pattern can easily be established by slightly disturbing the initial condition. However, all the flow patterns presented in this paper have been selected among the observed flow patterns characterized by a deviation towards the right, for the single purpose of facilitating comparisons between the different geometries. 
(a)
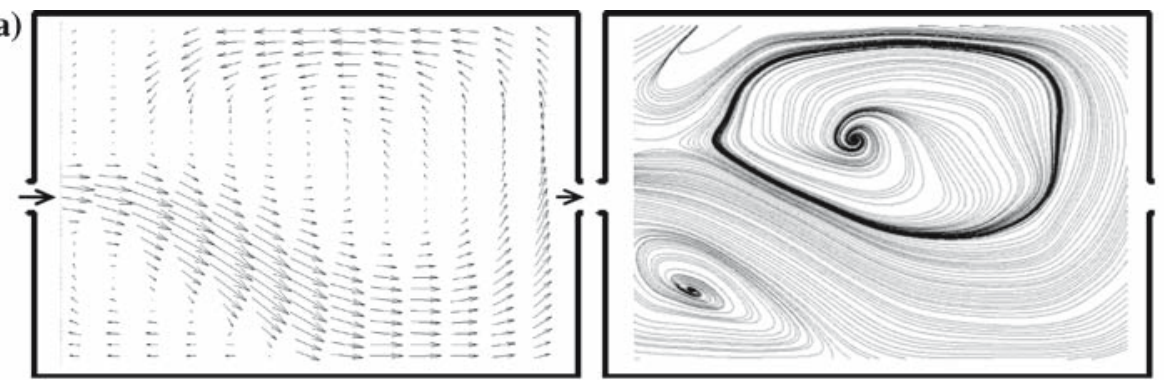

(b)

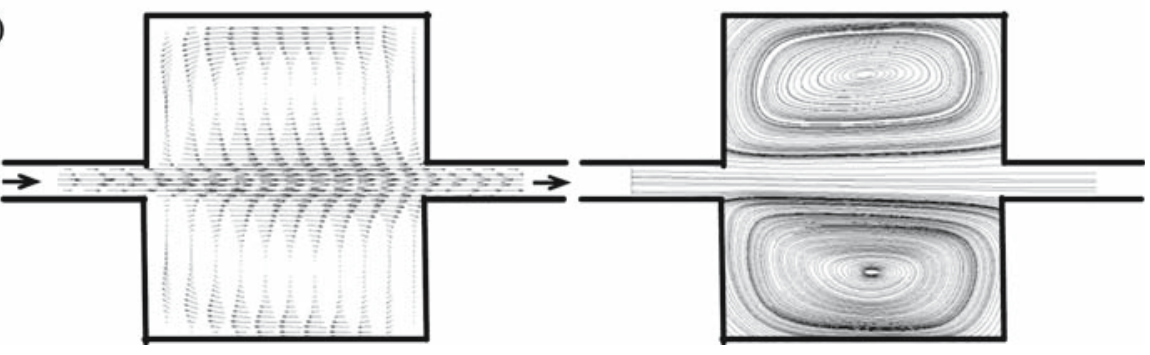

Fig. 2 Experimentally observed flow field and streamlines for geometries no. 1 (a) and no. 4 (b) [7]

The deflection of the jet can be explained by observing that a velocity increase on one side of the jet leads to a local reduced pressure, which in turn tends to amplify the deflection of the flow (Coanda effect) [20]. Simultaneously, in the deviated jet, an increase of the velocity leads to increased centrifugal forces, which tends to re-establish the symmetry of the flow pattern. A balance between those two effects is reached in the steady state.

For smaller basin widths or shorter basin lengths, as for instance in the case of test no. 4 (Fig. 2b), the flow remains essentially symmetric, with one circulation cell on each side of the centreline.

\section{Brief description of the numerical model WOLF 2D}

Depth-averaged flow simulations representing the experimental set-up have been performed with the numerical model WOLF 2D, developed at the University of Liege and based on an original finite volume scheme [16,17].

\subsection{Mathematical model}

The model is based on the two-dimensional depth-averaged equations of volume and momentum conservation, namely the "shallow-water" equations. In the "shallow-water" approach the only assumption states that velocities normal to a main flow direction are smaller than those in the main flow direction. As a consequence the pressure field is found to be almost hydrostatic everywhere. The large majority of flows occurring in rivers, even highly transient, can reasonably be seen as shallow, except in the vicinity of some singularities. In the present study, measured vertical velocity components have been verified to remain low compared to velocity components in the horizontal plane. Therefore the flow in the basin may be considered as shallow and thus mainly two-dimensional [9]. 
Simplified for a flat bottom, the depth-averaged equations of mass and momentum conservation [21] can be written as follows, using vector notations:

$$
\frac{\partial \vec{s}}{\partial t}+\frac{\partial \vec{f}}{\partial x}+\frac{\partial \vec{g}}{\partial y}+\frac{\partial \vec{f}_{\mathrm{d}}}{\partial x}+\frac{\partial \vec{g}_{\mathrm{d}}}{\partial y}=\vec{S}_{\mathrm{f}}
$$

with $\vec{s}=\left[\begin{array}{ll}h h u h v\end{array}\right]^{\mathrm{T}}$ the vector of the conservative unknowns. $\vec{f}$ and $\vec{g}$ represent the advective and pressure fluxes in directions $x$ and $y$, while $\vec{f}_{\mathrm{d}}$ and $\vec{g}_{\mathrm{d}}$ are the diffusive fluxes:

$$
\vec{f}=\left(\begin{array}{c}
h u \\
h u^{2}+\frac{1}{2} g h^{2} \\
h u v
\end{array}\right), \quad \vec{g}=\left(\begin{array}{c}
h v \\
h u v \\
h v^{2}+\frac{1}{2} g h^{2}
\end{array}\right), \quad \vec{f}_{\mathrm{d}}=-\frac{h}{\rho}\left(\begin{array}{c}
0 \\
\sigma_{x} \\
\tau_{x y}
\end{array}\right), \quad \vec{g}_{\mathrm{d}}=-\frac{h}{\rho}\left(\begin{array}{c}
0 \\
\tau_{x y} \\
\sigma_{y}
\end{array}\right) .
$$

$\vec{S}_{\mathrm{f}}$ designates the friction term:

$$
\vec{S}_{\mathrm{f}}=\left[\begin{array}{lll}
0 & \tau_{\mathrm{b} x} / \rho & \tau_{\mathrm{b} y} / \rho
\end{array}\right]^{\mathrm{T}} .
$$

The following notations have been used: $t$ represents the time, $x$ and $y$ the space coordinates, $h$ the water depth, $u$ and $v$ the depth-averaged velocity components, $g$ the gravity acceleration, $\rho$ the density of water, $\tau_{\mathrm{b} x}$ and $\tau_{\mathrm{b} y}$ the bottom shear stresses, $\sigma_{x}$ and $\sigma_{y}$ the turbulent normal stresses, and $\tau_{x y}$ the turbulent shear stress.

The bottom friction is conventionally modelled thanks to an empirical law, such as the Manning formula. The model enables the definition of a spatially distributed roughness coefficient. Besides, the friction along side walls is reproduced through a process-oriented formulation developed by the first, third and fifth authors [16]:

$$
\begin{aligned}
& \frac{\tau_{\mathrm{b} x}}{\rho}=g h u\left[\sqrt{u^{2}+v^{2}} \frac{n_{\mathrm{b}}^{2}}{h^{4 / 3}}+u \sum_{k_{x}=1}^{N_{x}} \frac{4}{3} \frac{n_{\mathrm{w}}^{2}}{h^{1 / 3} \Delta y}\right] \\
& \frac{\tau_{\mathrm{b} y}}{\rho}=g h v\left[\sqrt{u^{2}+v^{2}} \frac{n_{\mathrm{b}}^{2}}{h^{4 / 3}}+v \sum_{k_{y}=1}^{N_{y}} \frac{4}{3} \frac{n_{\mathrm{w}}^{3 / 2}}{h^{1 / 3} \Delta x}\right]
\end{aligned}
$$

where the Manning coefficient $n_{\mathrm{b}}$ and $n_{\mathrm{w}}\left(\mathrm{s} / \mathrm{m}^{1 / 3}\right)$ characterize, respectively, the bottom and the side-walls roughness. Those relations are particularized for Cartesian grids, as exploited in the present study. Values of $n_{\mathrm{b}}=0.01 \mathrm{~s} / \mathrm{m}^{1 / 3}$ and $n_{\mathrm{w}}=0.02 \mathrm{~s} / \mathrm{m}^{1 / 3}$ are used hereafter. The higher friction coefficient for the walls is justified by not-perfectly plane side-walls.

The turbulent stresses are expressed following the Boussineq's approximation (transposed for a depth-averaged model) [22,23]:

$$
\frac{\sigma_{x}}{\rho}=2\left(v+v_{\mathrm{T}}\right) \frac{\partial u}{\partial x}, \quad \frac{\sigma_{y}}{\rho}=2\left(v+v_{\mathrm{T}}\right) \frac{\partial v}{\partial y}, \quad \frac{\tau_{x y}}{\rho}=\frac{\tau_{y x}}{\rho}=\left(v+v_{\mathrm{T}}\right)\left(\frac{\partial u}{\partial y}+\frac{\partial v}{\partial x}\right),
$$

where $v$ represents the molecular kinematic viscosity, while the turbulent eddy viscosity $v_{\mathrm{T}}$ is computed by a turbulence closure model $\left(v \ll v_{\mathrm{T}}\right)$. For this purpose, two different approaches are compared in this study (see Subsect.4.2). First, a simple algebraic turbulence closure is adopted, assuming that the turbulence is bed-dominated. In such a case, the turbulent kinematic viscosity may be expressed as:

$$
v_{\mathrm{T}}=\alpha h u_{*},
$$


with $\alpha$ taking values of the order 0.5 [24]. Second, an original depth-averaged $k-\varepsilon$ model with two different length-scales accounting for vertical and horizontal turbulence mixing has been applied, as developed by Erpicum [17].

\subsection{Numerical implementation and boundary conditions}

The space discretization of the divergence form of the 2D conservative shallow-water equations (1) is performed by means of a finite volume scheme. For the applications considered in this study, this approach warranties that the numerical model is free from mass and momentum conservation errors. A Cartesian grid is exploited, with a cell size of $0.025 \mathrm{~m}$. Variable reconstruction at cells interfaces is performed linearly, in conjunction with slope limiting, leading to a second-order spatial accuracy.

Appropriate flux computation has always been a challenging issue in computational fluid dynamics. In the present study, fluxes $\vec{f}$ and $\vec{g}$ are computed by a Flux Vector Splitting (FVS) method developed by the first, third and fifth authors. Following this FVS, the upwinding direction of each term of the fluxes $\vec{f}$ and $\vec{g}$ is simply dictated by the sign of the flow velocity reconstructed at the cells interfaces. It has thus the advantage of being completely Froude independent and of facilitating a satisfactory adequacy with the discretization of the bottom slope term if present [17]. It can be formally expressed as follows:

$$
\vec{f}^{+}=\left(\begin{array}{c}
h u \\
h u^{2} \\
h u v
\end{array}\right) ; \quad \vec{f}^{-}=\left(\begin{array}{c}
0 \\
\frac{1}{2} g h^{2} \\
0
\end{array}\right) ; \quad \vec{g}^{+}=\left(\begin{array}{c}
h v \\
h u v \\
h v^{2}
\end{array}\right) ; \quad \vec{g}^{-}=\left(\begin{array}{c}
0 \\
0 \\
\frac{1}{2} g h^{2}
\end{array}\right)
$$

where the exponents + and - refer to, respectively, an upstream and a downstream evaluation of the corresponding terms. A Von Neumann stability analysis has demonstrated that this FVS leads to a stable spatial discretization of the terms $\partial \vec{f} / \partial x$ and $\partial \vec{g} / \partial y$ in Eq. 1 [16]. This FVS has already proved its validity and efficiency for numerous applications $[16,17,25$, $26]$. Due to their diffusive nature, the fluxes $\vec{f}_{\mathrm{d}}$ and $\vec{g}_{\mathrm{d}}$ are legitimately evaluated by means of a centred scheme.

Since the model is applied to compute steady-state solutions, the time integration is performed by means of a three-step first order accurate Runge-Kutta algorithm, providing adequate dissipation in time. For stability reasons, the time step is constrained by the CourantFriedrichs-Levy (CFL) condition based on gravity waves. A semi-implicit treatment of the bottom friction term (3) is used, without requiring additional computational costs.

The value of the specific discharge is prescribed as an inflow boundary condition. Besides, the transverse specific discharge is set to zero at the inflow. The outflow boundary condition is a constant water surface elevation: $0.2 \mathrm{~m}$. At solid walls, the component of the specific discharge normal to the wall is set to zero. For the purpose of evaluating the diffusive terms, the gradients of the unknowns must also be specified at the boundaries. These gradients in the direction parallel to the boundary are set to zero for simplicity, while the gradients of the variables in the direction normal to the boundary are properly evaluated by finite difference between the value at the boundary and the value at the centre of the adjacent cell [17].

\subsection{Other features of WOLF 2D}

The herein described model constitutes a part of the modelling system "WOLF", developed at the University of Liege. WOLF includes a set of complementary and interconnected modules for simulating free surface flows: process-oriented hydrology, 1D \& 2D hydrodynamic, sediment [16] or pollutant transport, air entrainment, as well as an optimisation tool (based 


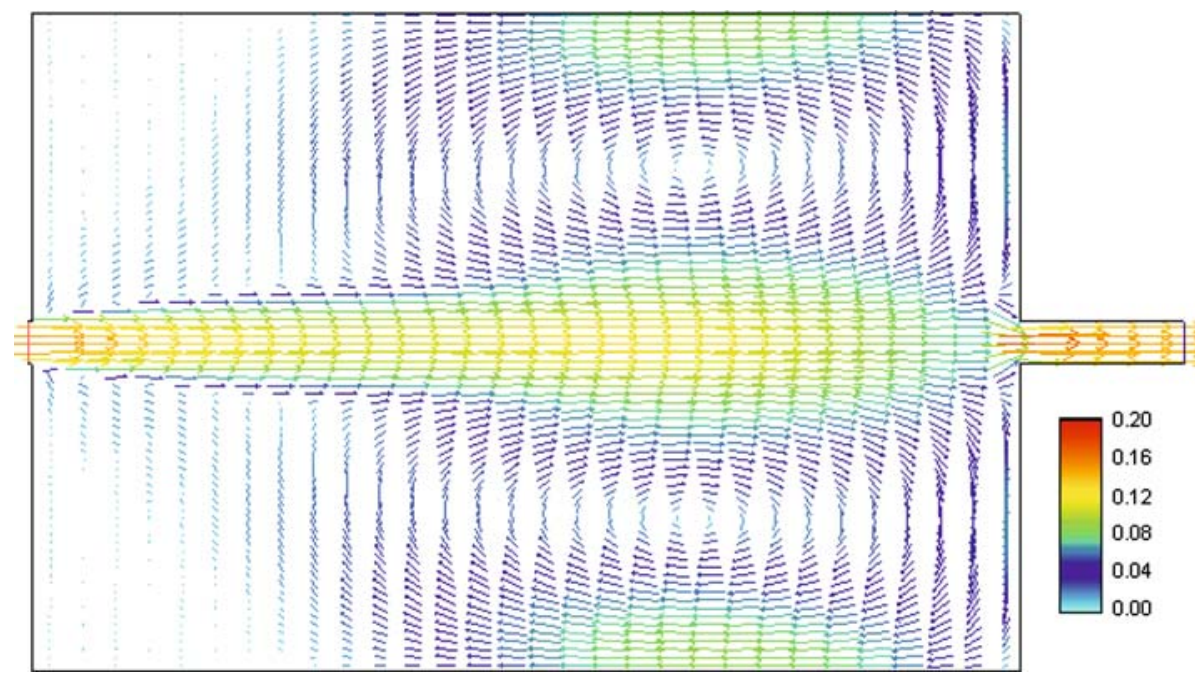

Fig. 3 Flow field simulated with a uniform specific discharge profile at inflow (algebraic turbulence closure). Velocity magnitude in $\mathrm{m} / \mathrm{s}$

on Genetic Algorithms) [17]. Other functionalities of WOLF 2D include the use of moment of momentum equations [16], the application of the cut-cell method [17], as well as computations considering vertical curvature effects by means of curvilinear coordinates in the vertical plane [25]. The model deals with multiblock [17] Cartesian grids and includes an automatics mesh refinement algorithm. A grid adaptation technique restricts the simulation domain to the wet cells. Besides, wetting and drying of cells is handled free of mass and momentum conservation errors [17].

\section{Numerical results and analysis}

\subsection{Ability of the model to represent the flow instability}

A first simulation has been performed based on the geometry and inflow/outflow conditions of the physical model. The algebraic turbulence model is used and the simulation is run until a steady-state flow field is reached. This obtained simulated flow field is perfectly symmetric (Fig. 3). Although not in agreement with experimental observations, this result was expected since neither the mathematical model nor the algorithm implementation are supposed to break the perfect symmetry of input data. Consequently, this first simulation result demonstrates that the model does not include any spurious numerical artefact tending to introduce dissymmetry in a problem with perfectly symmetric input data.

However, according to laboratory experiments, this symmetric flow field is not stable. Therefore, a second series of simulations has been undertaken with slightly disturbed distributions of the specific discharge at the inflow, in order to test the stability of the numerical solution. Instead of being uniform, the cross-sectional profile of the specific discharge is specified with a linear variation along the width of the inlet channel:

$$
q_{\text {in }}(y)=q_{0}+q_{1} 2 y / b,
$$



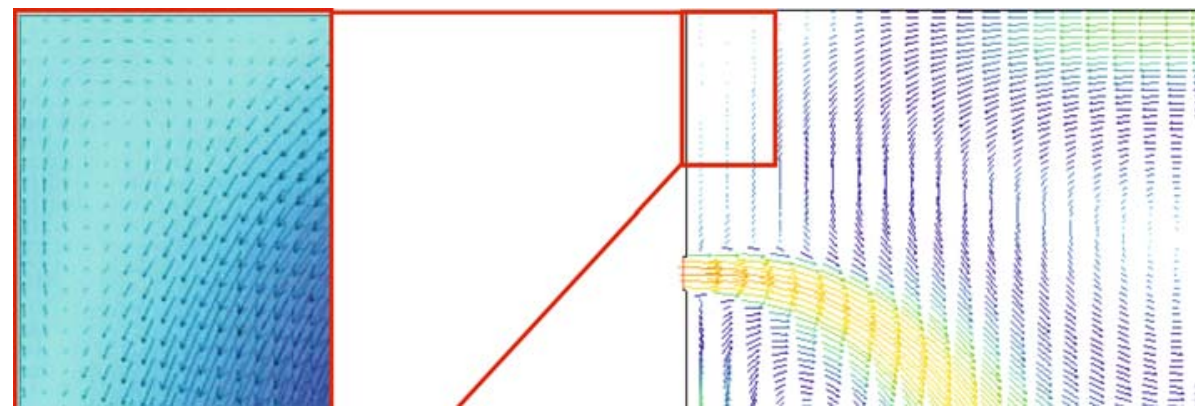

Fig. 4 Flow field simulated with a disturbed specific discharge profile at inflow (algebraic turbulence closure). Velocity magnitude in $\mathrm{m} / \mathrm{s}$

where $q_{\text {in }}\left(\mathrm{m}^{2} / \mathrm{s}\right)$ denotes the actual value specified as inflow boundary condition, $q_{0}\left(\mathrm{~m}^{2} / \mathrm{s}\right)$ is the reference value (total discharge divided by channel width) and $q_{1}\left(\mathrm{~m}^{2} / \mathrm{s}\right)$ measures the magnitude of the linear variation. $b(\mathrm{~m})$ designates the width of the inlet channel and $y(\mathrm{~m})$ is the transverse coordinate, varying between $-b / 2$ and $b / 2$.

As shown in Fig. 4, considering a minor change in the inflow boundary condition (with $q_{1} / q_{0}=2 \%$ ) leads to a totally different flow field. The output of the numerical model becomes globally consistent with experimental observations. Indeed, the deviation of the main jet is reproduced. The existence of three main vortices, as highlighted experimentally, is also predicted by the numerical model. The simulated reattachment length $L_{r 1}$ is $2.53 \mathrm{~m}$, which is less than $5 \%$ shorter than the experimental one of $2.65 \mathrm{~m}$. Note that neglecting the side-wall friction in identities (4) prevents the numerical model from reproducing the upstream left vortex (see in inset in Fig. 4).

The artificial disturbance of the flow introduced here, through the non-uniform profile of specific discharge, represents actually unavoidable small disturbances existing in the experimental set-up. The slight perturbation of the inflow has a particularly strong effect on the results because of the unstable nature of the symmetric flow field (see Sect.7). As a consequence, it can be concluded that the numerical model is able to reproduce the high sensitivity of the real flow to external disturbances and hence the unstable nature of the symmetric solution in the present configuration.

Besides, the jet can be deviated either towards the right or towards the left side of the basin. In other words, two stable solutions exist and either of them can be obtained depending on the sign of $q_{1}$ or on the sense of the deviation in the initial condition. The occurrence of two distinct stable flow fields is also confirmed by the experimental tests [7]. The similar existence of multiple solutions were reported previously by other authors [4].

It has been verified that, even with a uniform specific discharge profile as inflow boundary conditions, similar simulation results can also be obtained by starting the computation with a flow field initially deviated. In this case, the non-symmetric initial condition acts as a disturbance [4]. Nevertheless, this approach is not further explored in the present study.

Moreover, although the flow may deviate alternatively towards the right or towards the left, all simulation results presented in the present paper have been selected with the same deviation in order to make comparisons easier between the different geometries considered.

For comparing symmetric and non-symmetric results, a suitable quantitative indicator of the "intensity" of the non-symmetry of the flow field is introduced. It consists in evaluating the moment $m$ of the $u$-velocity field with respect to the centreline of the basin. The indicator 


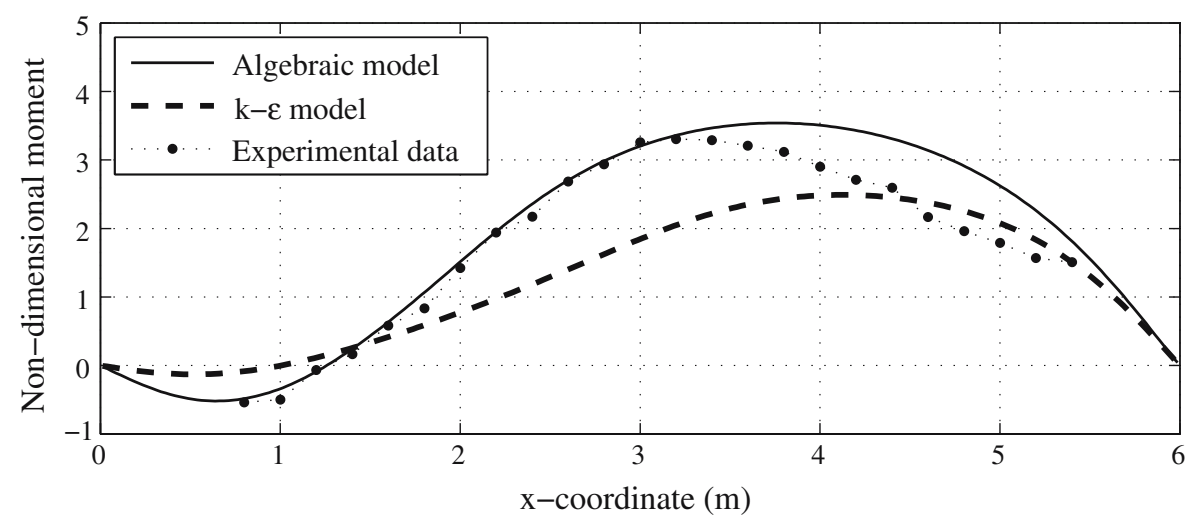

Fig. 5 Non-dimensional moment $m$ of the flow field in the rectangular basin of $6 \mathrm{~m}$ by $4 \mathrm{~m}$

is defined in non-dimensional form as follows:

$$
m(x)=\frac{1}{B} \int_{-B / 2}^{B / 2} \frac{u(x, y)-U}{U} \frac{2 y}{B} \mathrm{~d} y=\frac{1}{B} \int_{-B / 2}^{B / 2}\left(\frac{u}{U}-1\right) \frac{2 y}{B} \mathrm{~d} y=\frac{2}{U B^{2}} \int_{-B / 2}^{B / 2} u y \mathrm{~d} y
$$

where $U(\mathrm{~m} / \mathrm{s})$ is a reference velocity. The following value has been considered: $U=Q /\left(B h_{0}\right)$, where $h_{0}(\mathrm{~m})$ corresponds to the water depth at the downstream boundary condition, $Q\left(\mathrm{~m}^{3} / \mathrm{s}\right)$ is the total discharge and $B(\mathrm{~m})$ the basin width.

For any flow field which is symmetric with respect to the centreline, the moment remains equal to zero. For non-symmetric flow fields, the moment quantifies the deviation of the actual velocity profile compared to a symmetric one. For instance, $m=1$ if the velocity profile varies linearly between both side-walls of the basin with a difference between maximum velocity and mean velocity equal to three times the mean velocity.

In Fig. 5, the moment of the measured and simulated flow fields with either the algebraic or the $k-\varepsilon$ turbulence closure (see Subsect. 4.2 for a more comprehensive discussion) are compared. It can be seen that the sign of the moment provides information on the sense of rotation of the main vortices. In the upstream part of the basin, negative values of $m$ corresponds to an anti-clockwise rotating vortex, while in the rest of the basin, positive values of $m$ indicate a clockwise rotating vortex.

The moment $m$ characterizes the asymmetry of the flow field in any given cross-section. It can be further averaged along the basin length $L$, to provide a global quantitative indicator of non-symmetry of the flow in the whole computation domain (see Sect. 5):

$$
M=\frac{1}{L} \int_{0}^{L}|m| \mathrm{d} x .
$$

The absolute value of $m$ is used in order to prevent any compensation between values of opposite sign.

In order to verify that the simulated flow field does not significantly depend on the arbitrary value selected for the disturbance $q_{1}$, five simulations have been run with $q_{1} / q_{0}$ varying between 1 and 5\% (Table 2). In every case, the flow field remains very close to that one observed at the laboratory. For disturbance intensities between 1 and $5 \%$, simulated results 
Table 2 Length-averaged moment of the flow field for five different disturbance intensities at inflow

\begin{tabular}{llllllr}
\hline Disturbance $q_{1} / q_{0}$ & $0 \%$ & $1 \%$ & $2 \%$ & $3 \%$ & $4 \%$ & $5 \%$ \\
\hline Global moment $M(-)$ & 0.0000 & 1.9025 & 1.9057 & 1.9068 & 1.9082 & 1.9096 \\
\hline
\end{tabular}

present almost no effect $(<0.5 \%)$ of the disturbance intensity, as shown by the comparison of the global moment $M$ values in Table 2 .

\subsection{Detailed comparison between simulated and measured flow fields}

Figure 6 presents the measured and simulated $u$ component of the velocity in four different cross-sections in the basin.

Figure $6 \mathrm{a}, \mathrm{b}$ show that the algebraic turbulence closure tends to overestimate the velocity magnitude in the upstream right vortex, while the $k-\varepsilon$ model provides more accurate values of the velocity in this vortex. On the contrary, the main jet simulated by means of the $k-\varepsilon$ model remains too concentrated and the diffusion of the jet is better predicted by the algebraic turbulence closure. As mentioned previously, the simulation based on the algebraic turbulence closure leads to a satisfactory prediction of the main reattachment length. Figure $6 \mathrm{c}$ shows that, at cross-section $x=3 \mathrm{~m}$, the flow velocity computed by the $k-\varepsilon$ model is still reversed, while the experimental measurements confirm that the reattachment length is actually already exceeded. Indeed, the $k-\varepsilon$ model overestimates the reattachment length by $0.75 \mathrm{~m}$ (predicted value: $3.40 \mathrm{~m}$, instead of $2.65 \mathrm{~m}$ ). Finally, it must be noted that the algebraic turbulence closure predicts a small, however unrealistic, recirculation in the vicinity of the outlet channel, as can be seen on Fig. 4. The $k-\varepsilon$ model simulates a much more realistic flow field in this area.

It may be concluded that the numerical model captures at least qualitatively the global pattern of the complex flow field in the shallow reservoir. It reproduces both the main jet deflection and the development of the three main vortices. Moreover, the relative location and size of these vortices are predicted rather satisfactorily. Nevertheless, the prediction of the details of the flow field has still to be improved, as revealed by the comparison of velocity distributions in cross-sections. Indeed, the quantitative predictions of the depth-averaged velocity are found to deviate locally by up to $50 \%$, and even more in some regions. Some of these discrepancies are obviously also due to the fact that experimental data refer to surface velocity, while the simulated results are depth-averaged and are thus expected to take lower values.

\section{Influence of basin geometry on flow stability}

The herein presented comparison with experimental results focuses on the ability of the numerical model to predict the transition between stable and unstable configurations. For each specific geometry (see Table 1), the stability of a symmetric flow field is analysed by considering "quasi-symmetric" input data, as used in Subsect. 4.1. "Quasi-symmetric" input data means symmetric geometry, outflow conditions and initial conditions, but slightly disturbed inflow boundary condition, according to relation (8). A geometric configuration will be referred to as stable if a symmetric flow field remains stable for quasi-symmetric input data. On the contrary, the configuration will be said to be unstable if, for quasi-symmetric 

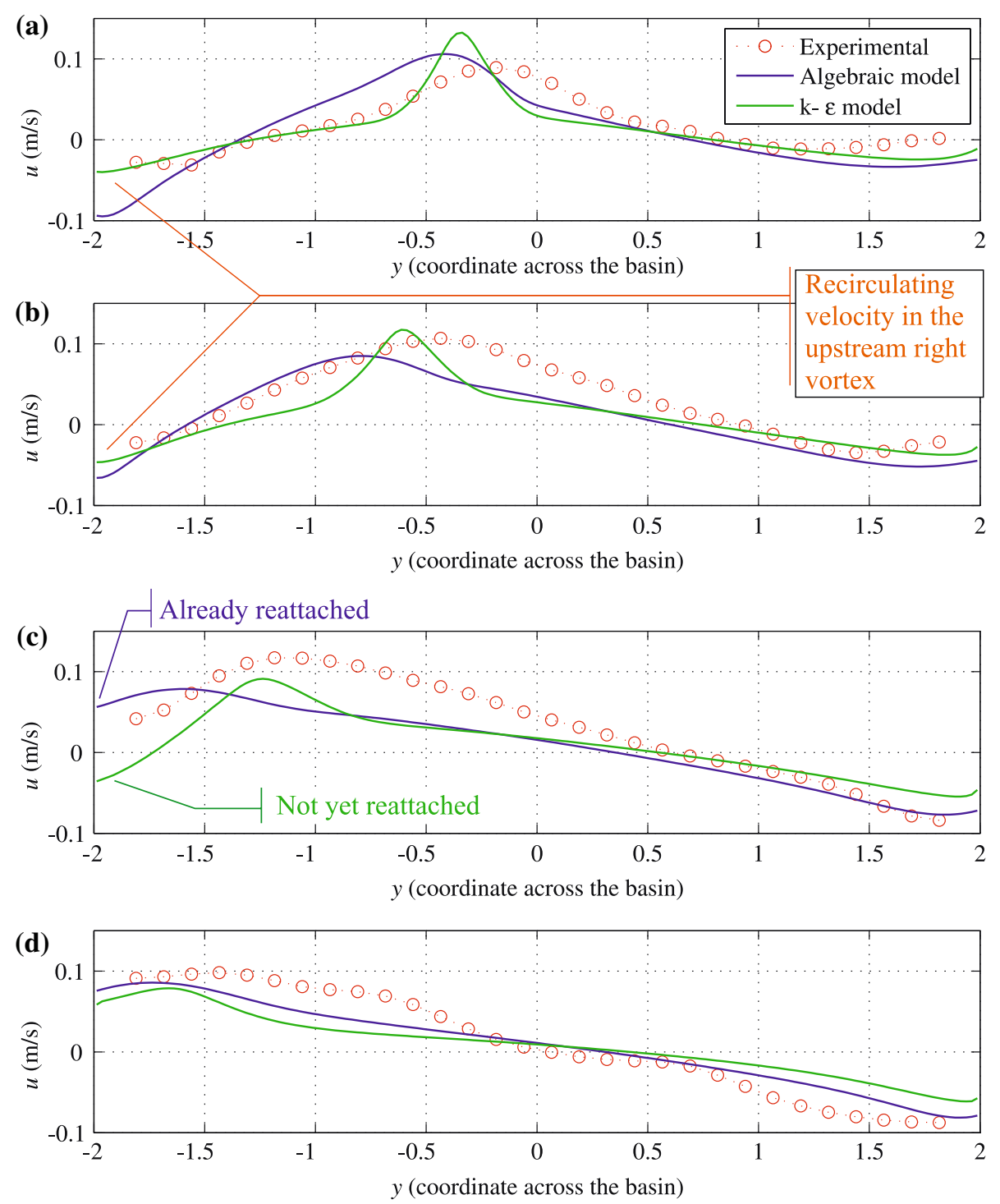

Fig. 6 Measured and simulated (with disturbance) velocity profiles in four cross-sections of the basin: (a) $x=1.5 \mathrm{~m}$, (b) $2 \mathrm{~m}$, (c) $3 \mathrm{~m}$ and (d) $4.5 \mathrm{~m}$ from the inlet

input data, a symmetric flow field becomes unstable and the actual steady solution deviates considerably from the symmetric one.

Figure 7 shows the measured and simulated flow fields for a constant basin width ( $4 \mathrm{~m})$ and a basin length reduced from 6 to $3 \mathrm{~m}$. For a length $L=5 \mathrm{~m}, 4 \mathrm{~m}$ and $3 \mathrm{~m}$ a stable symmetric flow field is present, while for $L=6 \mathrm{~m}$ this is not the case, as already shown in Fig. 4 . This classification between stable and unstable configurations obtained from the 2D numerical simulations is in agreement with experimental results.

Similarly, measured and simulated flow fields are compared in Fig. 8, for a constant basin length $(6 \mathrm{~m})$ but a varying basin width (in-between $B=0.5 \mathrm{~m}$ and $B=4 \mathrm{~m}$ ). 
(a)

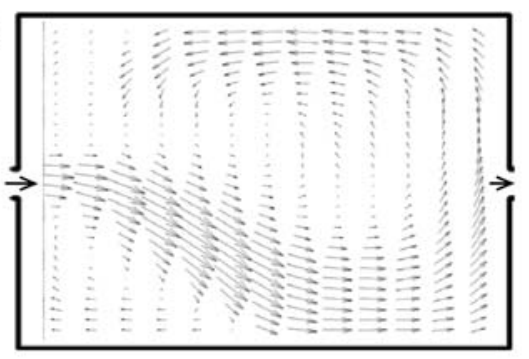

(b)

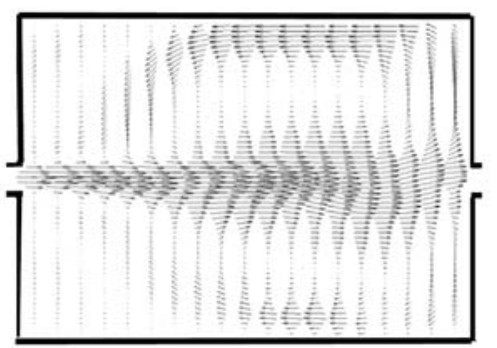

(c)

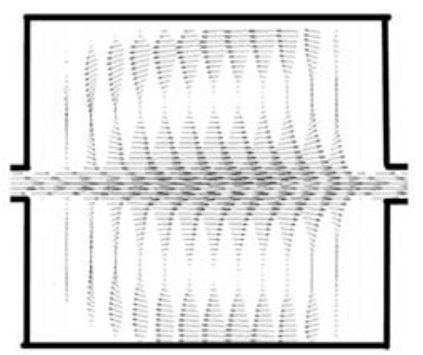

(d)

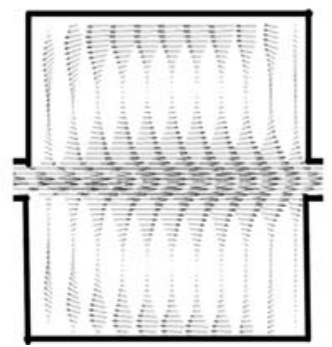

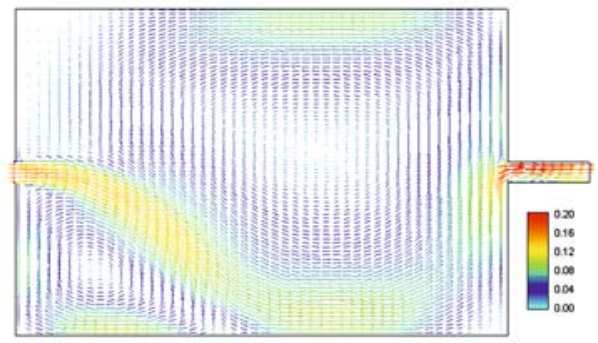
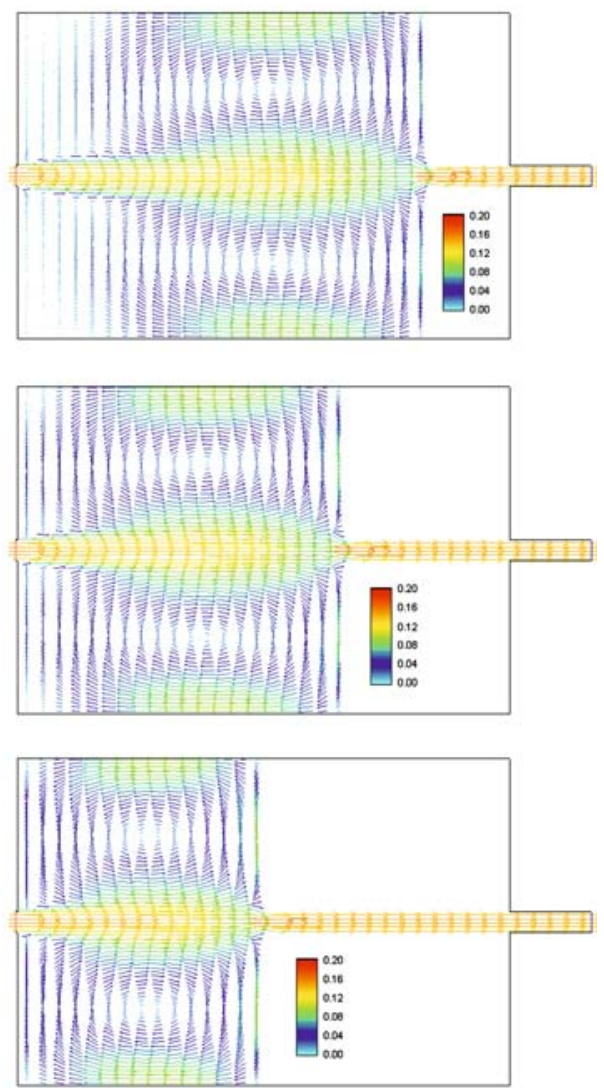

Fig. 7 Experimental (left) and simulated (right) flow fields in the basin $4 \mathrm{~m}$ wide and (a) $6 \mathrm{~m}$ long (test no. 1), (b) $5 \mathrm{~m}$ long (test no. 2), (c) $4 \mathrm{~m}$ long (test no. 3) and (d) $3 \mathrm{~m}$ long (test no. 4)

All configurations lead to a deviated flow, except the narrowest one, namely $B=0.5 \mathrm{~m}$. This conclusion is in agreement with experimental data, which means again that the numerical model performs well in identifying the stable and the unstable tested configurations.

Figure 9 summarizes the results obtained for the eight different geometries by showing the curve of the moment $m$ for each computed flow field (solid lines). The four non-trivial curves correspond to the four different basin widths and to the single basin length which lead to an instability of a symmetric flow field, whereas all stable configurations are represented by a uniform zero-value of $m$, which is consistent with the role of $m$ as an indicator of non-symmetry. Experimental data are also reproduced in Fig. 9 (dotted lines), recalling that 
(a)

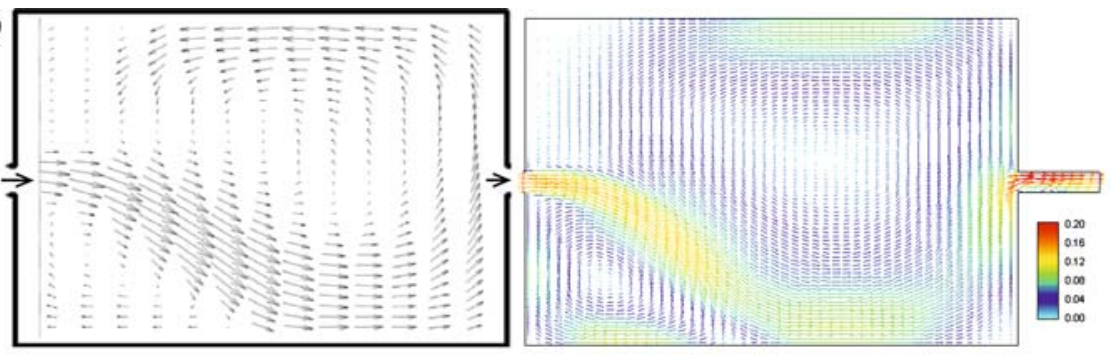

(b)

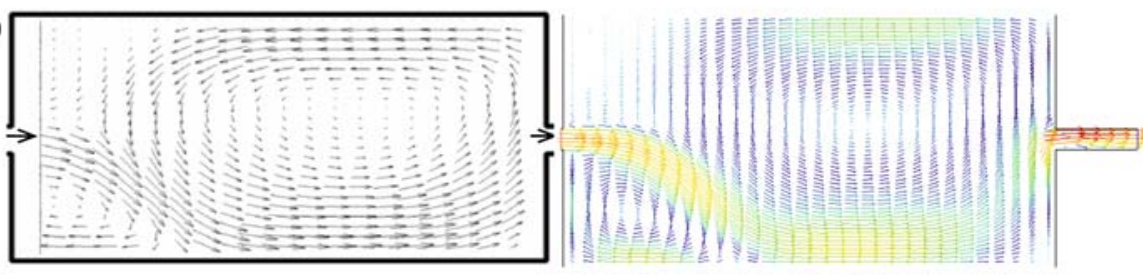

(c)

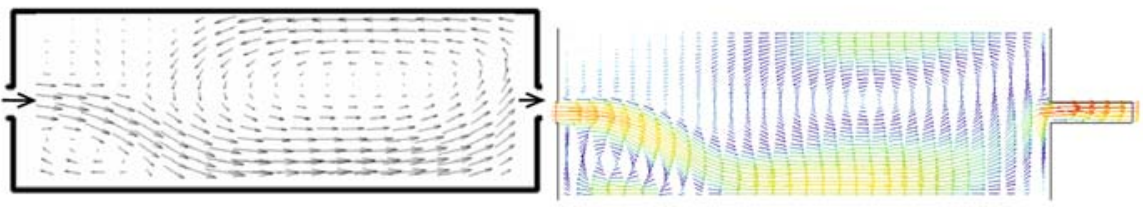

(d)

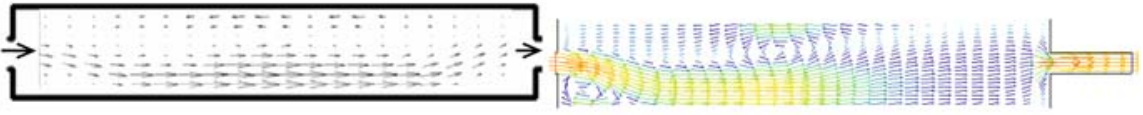

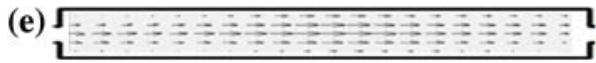

Fig. 8 Experimental (left) and simulated (right) flow fields in the basin $6 \mathrm{~m}$ long and (a) $4 \mathrm{~m}$ wide (test no. 1), (b) $3 \mathrm{~m}$ wide (test no. 5), (c) $2 \mathrm{~m}$ wide (test no. 6), (d) $1 \mathrm{~m}$ wide (test no. 7) and (e) $0.5 \mathrm{~m}$ wide (test no. 8)

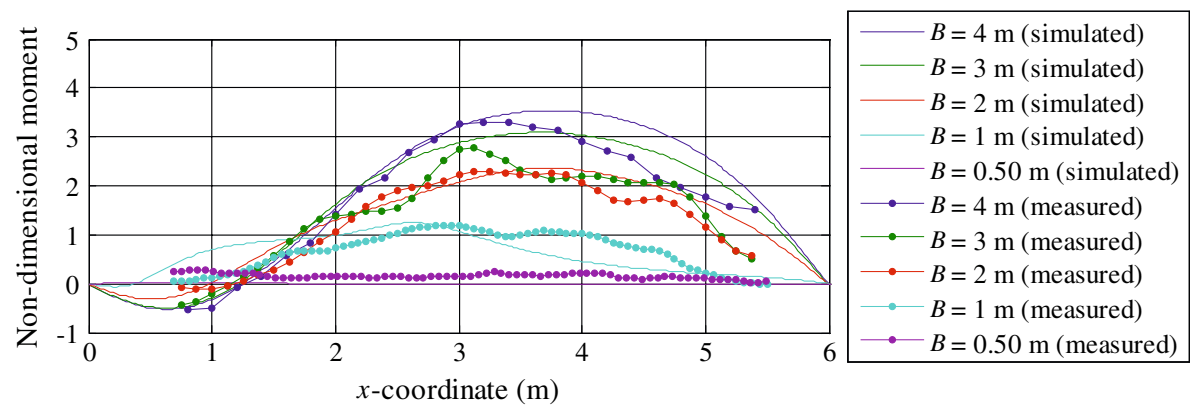

Fig. 9 Non-dimensional moment $m$ of the flow field in the rectangular basin of varying width. Numerical results (solid lines) and experimental data (dotted lines) are reproduced

the numerical model succeeds in simulating the global pattern of the flow but still requires enhancements to achieve a satisfactory quantitative agreement with measurements.

The moment $m$ plotted in Fig. 9 is a preliminary step to evaluate the length-averaged moment $M$, as defined by relation (10), in order to illustrate the bifurcating behaviour of the flow field in the studied shallow basins. 

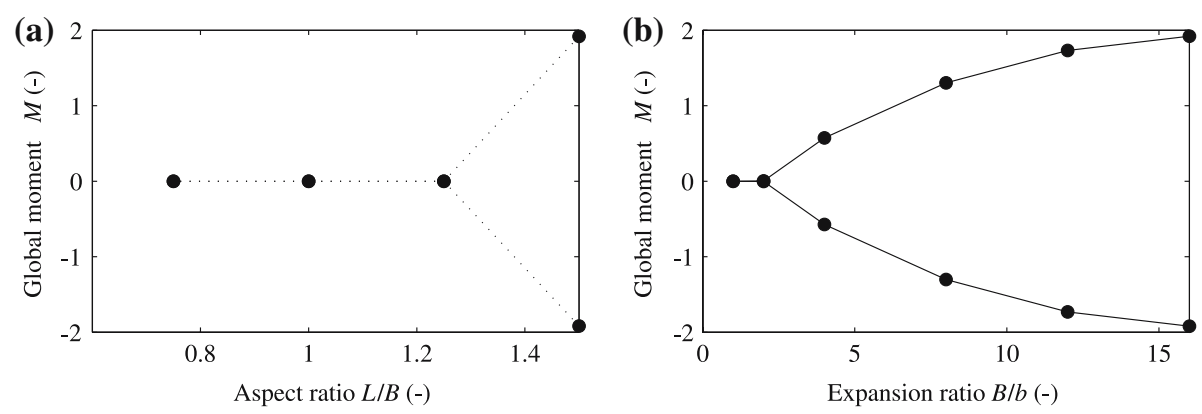

Fig. 10 Bifurcation diagram showing the global moment $M$ as a function of different aspect ratios (a, for varying basin length) and of different expansion ratios (b, for varying basin width)

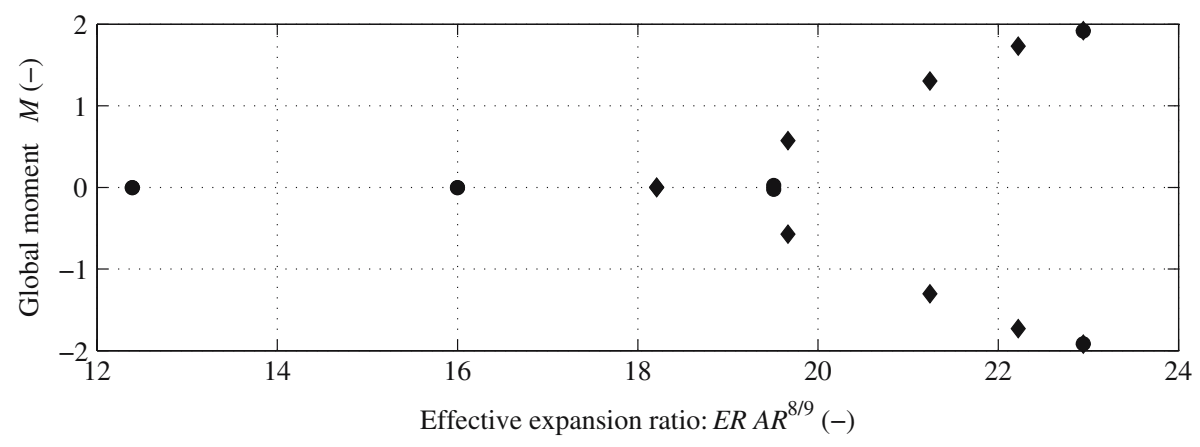

Fig. 11 Bifurcation diagram showing the mean moment $M$ as a function of the combined indicator $R$. Circles correspond to tests with varying length and diamonds to tests with varying width

A bifurcating solution is a solution which can branch (bifurcate) from a given basic solution when the basic solution loses its stability with respect to disturbances. Moreover, once a bifurcation takes place, at least one symmetry property of the base flow solution is broken [27]. Such a bifurcation phenomenon is dependent on a controlling parameter $R$, which in the present case is either one of the two geometric ratios $E R$ or $A R$ defined in Sect. 2. The flow solution is said to bifurcate from the base flow solution at $R=R_{\mathrm{c}}$ if for $R>R_{\mathrm{c}}$ there are at least two solutions which merge with the base flow solution for $R<R_{\mathrm{c}}$ [27]. Indeed, in the present case, the non-symmetric solutions can be equally obtained on one side or on the other one. In Fig. 10, the global moment $M$ is plotted as a function of the aspect ratio $A R$ for the different tested basin lengths (a) and as a function of the expansion ratio $E R$ for the different basin widths considered (b).

In order to represent all global moments in a single bifurcation diagram, a single controlling parameter $R$, combining $E R$ and $A R$, has been identified, in such a way that a threshold value $R_{\mathrm{c}}$ delineates all symmetric cases $\left(R<R_{\mathrm{c}}\right)$ of all non-symmetric ones $\left(R>R_{\mathrm{C}}\right)$. If each geometry is represented by one point in the plane $(A R, E R)$, this plane can be divided into two regions: the first one containing the points representing stable configurations and the second one corresponding to unstable configurations. The marginal stability curve, delineating the boundary between both sets of points, can be mathematically approximated by the following function: $E R \simeq(0.035 A R)^{-8 / 9}$. As a result, $R=E R \times A R^{8 / 9} \approx E R \times A R^{0.89}$ may be used as the controlling parameter to plot the global bifurcation diagram, as displayed by Fig. 11. The precise formulation of the indicator $R$ should of course be confirmed based 


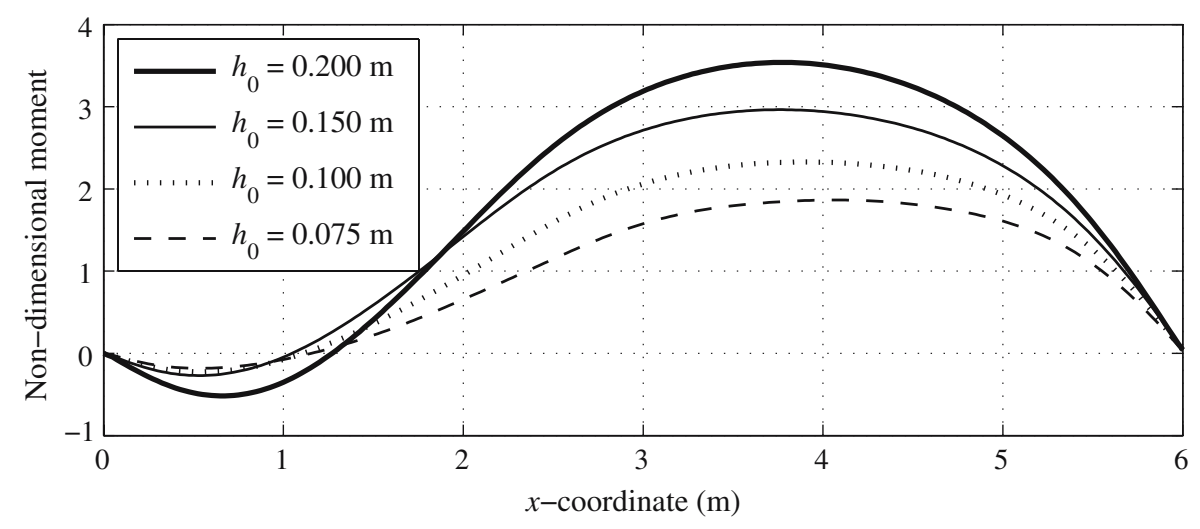

Fig. 12 Non-dimensional moment $m$ of the flow field in the rectangular basin of $6 \mathrm{~m}$ by $4 \mathrm{~m}$ for four different water depths as downstream boundary conditions

on additional experimental tests or numerical simulations, dedicated to refining the interval in which each bifurcation apex is located (see Fig. 10).

\section{Influence of hydraulic conditions on flow stability}

Finally, the influence of the hydraulic conditions on the stability of the flow in the original basin $(6 \mathrm{~m}$ by $4 \mathrm{~m})$ has been appreciated by varying the water depth between 0.075 and $0.200 \mathrm{~m}$ (Table 3). Figure 12 shows the non-dimensional moment $m$ for the four different hydraulic conditions considered and reveals that the jet deviation tends to be reduced for decreasing water depth. Indeed, due to the higher flow velocity, the stabilizing effect of bed friction is amplified when the water depth is reduced. The relative importance of bottom friction forces compared to turbulent diffusion can be evaluated by the product of the bottom friction parameter $S$, defined as $S=(b / 2 h)\left(g n^{2} / h^{1 / 3}\right)$ in the case of the Manning friction law, and the turbulent Reynolds number $\operatorname{Re}_{\mathrm{T}}$ (based on the turbulent eddy viscosity). As shown in Table 3, the product $S \times R e_{\mathrm{T}}$ is increased by almost one order of magnitude when the water depth is varied from 0.020 to $0.075 \mathrm{~m}$. Nevertheless, the flow field remains significantly non-symmetric in all four tested cases. This observation is confirmed by the values of the global moment $M$ displayed in Table 3.

\section{Theoretical analysis of the flow stability}

The linear stability analysis provides an indication on whether a particular base flow is stable or unstable and can be used to describe the structure of the critical motion which takes place just above the threshold [10]. Mathematically, the problem consists in predicting the evolution of an arbitrary disturbance assumed to be initially superposed on the symmetric base flow. If the disturbance vanishes for $t \rightarrow \infty$, the flow field is regarded as stable, otherwise it is deemed as unstable [28]. In order to predict the behaviour of the disturbance, one assumes that the disturbance is small enough, so that the equations governing their evolution may be linearized. Such an approach is adequate because the interest is focused on the onset of a possible instability of a given symmetric base flow [28]. 
Table 3 Tested water depths as downstream boundary condition and corresponding non-dimensional numbers as well as global moment

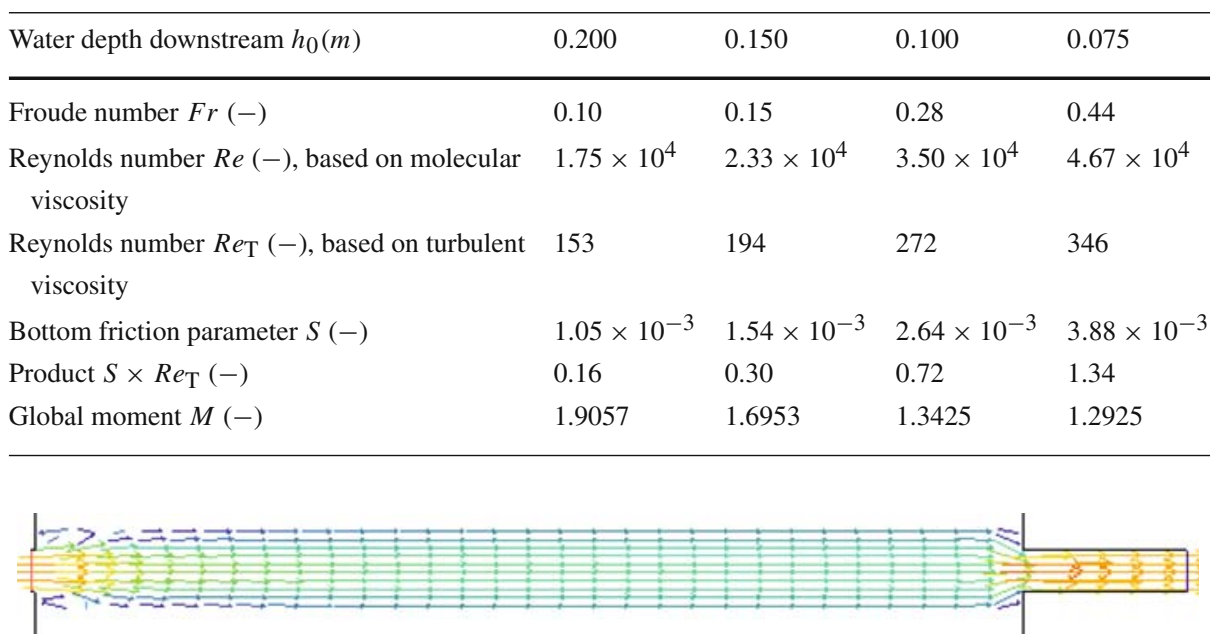

Fig. 13 Flow field simulated with a uniform specific discharge profile at inflow for the $3 \mathrm{~m}: 4 \mathrm{~m}$ basin (algebraic turbulence closure). Velocity magnitude in $\mathrm{m} / \mathrm{s}$

Two distinct base flows are considered, namely the symmetric undisturbed solutions in the $6 \mathrm{~m}$ by $4 \mathrm{~m}$ basin (see test no. 1 in Table 1 and Fig. 3) and in the $6 \mathrm{~m}$ by $0.5 \mathrm{~m}$ basin (see test no. 8 in Table 1 and Fig. 13).

\subsection{Governing equation for small perturbations}

The rigid-lid approximation is adopted for the linear stability analysis. This assumption is valid for low Froude numbers $(F r<0.6 \sim 0.7)$, as revealed by computations performed by Ghidaoui and Kolyshkin $(1999,2003)$ [2,10], which is verified in the present case: $F r \sim 0.1$. In such conditions, motions of interest are not associated to gravity waves and the rigid-lid approximation is hence appropriate. Moreover, water depths are quasi constant in the basin. Indeed their average value is $0.201 \mathrm{~m}$, with a standard deviation below $10^{-4}$. As a result of the rigid-lid assumption, it will be possible to reduce the governing system of equations for disturbances to a single scalar equation. For a constant water depth, the rigid-lid form of the shallow-water equations (1) can be written as follows [1,3]:

$$
\begin{gathered}
\frac{\partial u}{\partial x}+\frac{\partial v}{\partial y}=0 \\
\frac{\partial u}{\partial t}+u \frac{\partial u}{\partial x}+v \frac{\partial u}{\partial y}=-\frac{1}{\rho} \frac{\partial p}{\partial x}-\frac{g n_{\mathrm{b}}^{2}}{h^{4 / 3}} u \sqrt{u^{2}+v^{2}}+v_{\mathrm{T}}\left(\frac{\partial^{2} u}{\partial x^{2}}+\frac{\partial^{2} u}{\partial y^{2}}\right), \\
\frac{\partial v}{\partial t}+u \frac{\partial v}{\partial x}+v \frac{\partial v}{\partial y}=-\frac{1}{\rho} \frac{\partial p}{\partial y}-\frac{g n_{\mathrm{b}}^{2}}{h^{4 / 3}} v \sqrt{u^{2}+v^{2}}+v_{\mathrm{T}}\left(\frac{\partial^{2} v}{\partial x^{2}}+\frac{\partial^{2} v}{\partial y^{2}}\right),
\end{gathered}
$$

where the wall-roughness is neglected and the turbulent kinematic viscosity is considered as constant. 

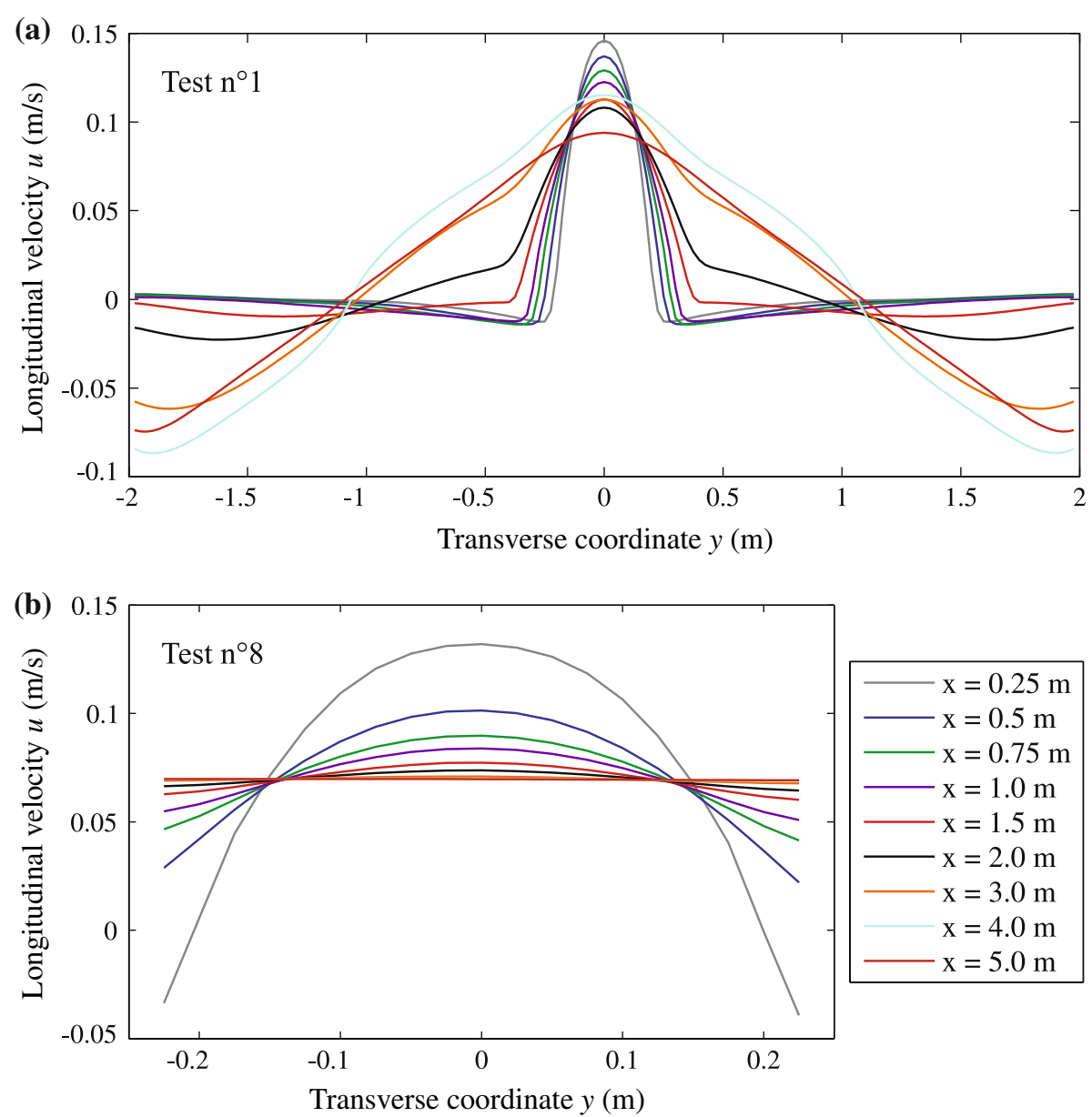

Fig. 14 Transverse profiles of the longitudinal velocity computed for test no. $1(6 \mathrm{~m}$ by $4 \mathrm{~m})$ and test no. 8 $(6 \mathrm{~m}$ by $0.5 \mathrm{~m})$

Several authors have shown that the shape of the transverse profile of the longitudinal velocity has a prevailing influence on the stability characteristics of shallow flows [10]. Therefore, in the following, the stability of the $u$ velocity profile in a number of cross-sections is investigated (see Fig. 14).

Hence, the base flow is assumed to have the following structure:

$$
u=u_{0}(y), \quad v=0, \quad p=p_{0}(x) .
$$

The pressure field $p_{0}$ verifies the longitudinal momentum conservation:

$$
\frac{\partial p_{0}}{\partial x}=-\frac{g n_{\mathrm{b}}^{2}}{h^{4 / 3}} u_{0}^{2}+v_{\mathrm{T}}\left(\frac{\partial^{2} u_{0}}{\partial y^{2}}\right)=G,
$$

where $G$ is assumed not to depend on $y$ (see Chu et al. 1991 [3]).

The selection of the plane parallel base flow (14) constitutes obviously a first approximation, since both the streamwise velocity gradients and the transverse velocities are neglected. 
However, even such a simple approach will provide some valuable results on the relative stability of the different tested configurations.

Substituting (14) into Eqs. 11-13 and linearizing the equations in the neighbourhood of the base flow, the following system of ordinary differential equations for the disturbances $u^{\prime}$, $v^{\prime}$ and $p^{\prime}$ is obtained:

$$
\begin{gathered}
\frac{\partial u^{\prime}}{\partial x}+\frac{\partial v^{\prime}}{\partial y}=0 \\
\frac{\partial u^{\prime}}{\partial t}+u_{0} \frac{\partial u^{\prime}}{\partial x}+v^{\prime} \frac{\partial u_{0}}{\partial y}=-\frac{1}{\rho} \frac{\partial p^{\prime}}{\partial x}-2 \frac{g n_{\mathrm{b}}^{2}}{h^{4 / 3}} u_{0} u^{\prime}+v_{\mathrm{T}}\left(\frac{\partial^{2} u^{\prime}}{\partial x^{2}}+\frac{\partial^{2} u^{\prime}}{\partial y^{2}}\right), \\
\frac{\partial v^{\prime}}{\partial t}+u_{0} \frac{\partial v^{\prime}}{\partial x}=-\frac{1}{\rho} \frac{\partial p^{\prime}}{\partial y}-\frac{g n_{\mathrm{b}}^{2}}{h^{4 / 3}} u_{0} v^{\prime}+v_{\mathrm{T}}\left(\frac{\partial^{2} v^{\prime}}{\partial x^{2}}+\frac{\partial^{2} v^{\prime}}{\partial y^{2}}\right),
\end{gathered}
$$

with the following boundary conditions at $y= \pm B / 2$ :

$$
v^{\prime}=0 \text { and } \frac{\partial v^{\prime}}{\partial y}=0 .
$$

By combining those three equations, a single scalar equation for the transverse velocity $v^{\prime}$ can be obtained, the solution of which is simpler than a direct integration of the complete system.

If one differentiates (17) with respect to $y,(18)$ with respect to $x$, and takes the difference, the pressure drops out and, after differentiating once more with respect to $x$ and introducing (16) to eliminate $u^{\prime}$, the following result is obtained:

$$
\begin{aligned}
& u_{0}\left(\frac{\partial^{3} v^{\prime}}{\partial x \partial y^{2}}+\frac{\partial^{3} v^{\prime}}{\partial x^{3}}\right)+\frac{\partial^{3} v^{\prime}}{\partial t \partial y^{2}}+\frac{\partial^{3} v^{\prime}}{\partial t \partial x^{2}}-\frac{\partial v^{\prime}}{\partial x} \frac{\mathrm{d}^{2} u_{0}}{\mathrm{~d} y^{2}} \\
& \quad=-\frac{g n^{2}}{h^{4 / 3}}\left(2 \frac{\partial v^{\prime}}{\partial y} \frac{\mathrm{d} u_{0}}{\mathrm{~d} y}+2 u_{0} \frac{\partial^{2} v^{\prime}}{\partial y^{2}}+u_{0} \frac{\partial^{2} v^{\prime}}{\partial x^{2}}\right)+v_{\mathrm{T}}\left(\frac{\partial^{4} v^{\prime}}{\partial x^{4}}+2 \frac{\partial^{4} v^{\prime}}{\partial x^{2} \partial y^{2}}+\frac{\partial^{4} v^{\prime}}{\partial y^{4}}\right)
\end{aligned}
$$

Following the conventional approach for linear stability analysis, the small-amplitude disturbances superimposed on the base flow are harmonic in $x, t[1,3]$ :

$$
v^{\prime}=\phi_{v}(y) e^{i k(x-c t)}
$$

in which $\phi_{v}(\mathrm{~m} / \mathrm{s})$ is a complex amplitude function, $k(\mathrm{rad} / \mathrm{m})$ is the wave number of the disturbance and $c(\mathrm{~m} / \mathrm{s})$ has real and imaginary parts: $c=c_{\mathrm{r}}+i c_{\mathrm{i}}$. The real part $c_{\mathrm{r}}$ is the wave velocity and the imaginary part $c_{\mathrm{i}}$ multiplied by $k$ is the amplification rate of the disturbance. The boundary conditions (19) on the velocity disturbance $v^{\prime}$ imply similar specifications on the disturbance amplitude at $y= \pm B / 2: \phi_{v}=0$ and $\partial \phi_{v} / \partial y=0$.

The values of $c$ are determined by an eigenvalue problem (see below): $c_{s}=c_{\mathrm{r} s}+i c_{\mathrm{i} s}$, $s=1,2, \ldots$ and they control the linear stability of the base flow described by (14). This base flow is said to be linearly stable if $c_{\mathrm{is}}<0$ for all $s$ and $k$, and linearly unstable otherwise. 
Introducing (21), one obtains the following ordinary differential equation:

$$
\begin{aligned}
& \left(u_{0}-c\right)\left(\frac{\mathrm{d}^{2} \phi_{v}}{\mathrm{~d} y^{2}}-k^{2} \phi_{v}\right)-\frac{\mathrm{d}^{2} u_{0}}{\mathrm{~d} y^{2}} \phi_{v} \\
& \quad=-\xi\left(-k^{2} \phi_{v}+2 \frac{\mathrm{d}^{2} \phi_{v}}{\mathrm{~d} y^{2}}+\frac{2}{u_{0}} \frac{\mathrm{d} u_{0}}{\mathrm{~d} y} \frac{\mathrm{d} \phi_{v}}{\mathrm{~d} y}\right)+\frac{\nu_{\mathrm{T}}}{i k}\left(\frac{\mathrm{d}^{4} \phi_{v}}{\mathrm{~d} y^{4}}-2 k^{2} \frac{\mathrm{d}^{2} \phi_{v}}{\mathrm{~d} y^{2}}+k^{4} \phi_{v}\right),
\end{aligned}
$$

where the friction parameter $\xi$ is defined as follows:

$$
\xi=\frac{g n^{2}}{h^{4 / 3}} \frac{u_{0}}{i k}
$$

Equation 22 is the modified Orr-Sommerfeld equation, as derived by van Prooijen et al. (1997) [29], particularized to the Manning formula for bed friction. Equation 22 together with the boundary conditions (19), implying that $\phi_{v}=0$ and $\partial \phi_{v} / \partial y=0$ at $y= \pm B / 2$, form an eigenvalue problem. If the turbulent viscosity is set to zero, equation (22) is known as the Rayleigh equation modified to account for bed friction.

\subsection{Resolution of the governing equations for disturbances}

A number of techniques are reported in the literature for solving (22), such as the pseudospectral collocation methods based on Chebyshev polynomials $[2,30]$. Here, the numerical procedure for the solution of the eigenvalue problem is based on a finite difference scheme [29].

Prior to numerically solve equation (22), an analytical approximation of the solution is sought. Although rather crude, this analytical approach provides a preliminary insight into the relative strength of the terms having a damping effect on perturbations for the two considered base flows.

As a first approximation, $\phi_{v}(y)$ is assumed to be harmonic with respect to $y: \phi_{v}=\Phi e^{i l y}$, where $l$ is the wave number in the $y$ direction. Under such condition, Eq. 22 leads to the following expression for $k c_{\mathrm{i}}$, which highlights here the contribution of the two damping effects in the system, namely friction and turbulent diffusion:

$$
k c_{\mathrm{i}}=-\frac{g n^{2}}{h^{4 / 3}} u_{0} \frac{2 l^{2}+k^{2}}{l^{2}+k^{2}}-v_{\mathrm{T}}\left(l^{2}+k^{2}\right) .
$$

Dimensionally, the above expression may be considered as the inverse of a characteristic time $\tau$, the value of which is straightforward to evaluate based on the algebraic closure (6) and if values of $k$ and $l$ are assumed to correspond, for instance, to the fundamental mode: $k=\pi / L$ and $l=\pi / B$. The values of $\tau$ corresponding to the two considered base flows, namely $\tau_{1}$ and $\tau_{8}$ referring, respectively, to test no. 1 and test no. 8 , may be compared: $\tau_{1} / \tau_{8} \simeq 3.3$, revealing that the "damping rate" $\left|k c_{\mathrm{i}}\right|$ is above three times higher in the case of Test 8 and the damping of perturbations is hence correspondingly stronger.

Second, a finite difference technique has been used to solve the eigenvalue problem associated to Eq. 22 in the domain defined by: $-B / 2 \leq y \leq B / 2$. In a first approximation, the modified Rayleigh equation is considered. The base flows $u_{0}(y)$ are velocity profiles (see Fig. 14) extracted from the symmetric flow fields illustrated in Figs. 3, 13, which result from 2D computations by means of the above-mentioned 2D finite volume model (see Sect. 3). Therefore the same mesh size is kept for the finite difference grid. Nodes are placed at the boundaries $y= \pm B / 2$, to enable easy specification of the remaining boundary condition: 


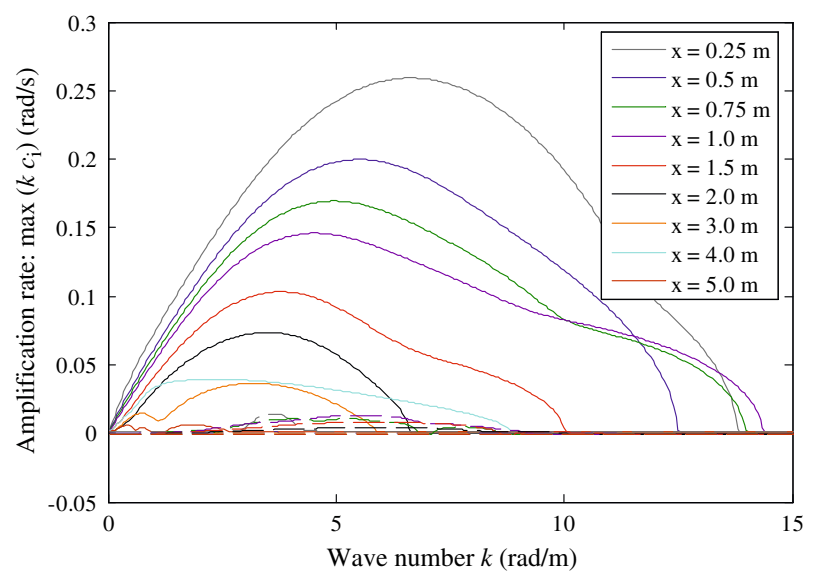

Fig. 15 Wave amplification rate: $\max _{s}\left(k c_{\mathrm{is}}\right)(\mathrm{rad} / \mathrm{s})$, as a function of the wave number $k(\mathrm{rad} / \mathrm{m})$, computed for velocity profiles corresponding to test no. 1 (solid lines) and test no. 8 (dashed lines) in nine different cross-sections located at a distance $x$ from the inlet

$\phi_{v}=0$ on the walls. Based on centred finite differences, the derivatives of $\phi_{v}$ and $u_{0}$ involved in Eq. 22 are discretized. The resulting symbolic expression can formally be written as:

$$
(\mathbf{A}-c \mathbf{B}) \vec{\phi}=0
$$

where $\vec{\phi}$ designates the vector of the approximations of $\phi_{v}$ at the nodes of the finite difference grid.

Non-trivial solutions of (25) are only possible if $\operatorname{det}(\mathbf{A}-c \mathbf{B})=0$. This constraint leads to a spectrum of eigenvalues $c_{s}$ for each wave number $k$. Figure 15 shows the wave amplification rate $\max _{s}\left(k c_{\text {is }}\right)$ as a function of the wave number for nine different velocity profiles identified by their longitudinal coordinate $x$. In the case of test no. 1, for all velocity profiles except one $(x=5 \mathrm{~m})$, the amplification rate is significantly higher than zero in a wide range of wave numbers, revealing that the symmetric flow field is definitely unstable in this geometric configuration. This result is consistent with experimental observations (Fig. 2) and 2D simulations (Fig. 4).

For test no. $8, c_{\text {is }}$ takes negative values for several velocity profiles and remains in all cases much smaller than for test no. 1. Maximum amplification rates are about 20 times smaller for test no. 8 than for test no. 1, revealing a far less unstable symmetric flow field for the geometric configuration of test no. 8 than in test no. 1. This is again in agreement with both experimental data and with the computed flow field (see Fig. 8). The remaining non-negative values of $c_{\text {is }}$ are most probably explained by the rather crude approximation (14) taken for the base flow and the fact that one consequently neglects the truly two-dimensional features of the actual base flow in the basin with the sudden expansion and contraction $\left(u_{0}(x, y)\right.$, $\left.v_{0}(x, y) \neq 0\right)$.

\section{Summary and conclusions}

The present paper presents numerical and theoretical analyses of shallow flows in a series of rectangular basins with experimental data. The experimental results include flow visu- 
alization for a constant discharge but for eight different basin geometries (varying length and varying width of the basin). Systematically the length and width of the basin have been decreased starting from a $6 \mathrm{~m}$ long and $4 \mathrm{~m}$ wide basin. They show that, in spite of the geometrically and hydraulically symmetric setup with respect to the centreline of the basin, non-symmetric flow fields are observed for certain geometric and hydraulic conditions, while the flow field remains symmetric in narrower or shorter basins. In the case of non-symmetric flow patterns, three main vortices can be identified in the flow field.

Numerical modelling has been performed with the conservative finite volume algorithm WOLF 2D, solving the shallow-water equations. Both an algebraic turbulence closure and a $k-\varepsilon$ model are exploited and compared. For symmetric input data, the numerical model provides also a perfectly symmetric result. However, if the inflow boundary condition is disturbed by a slightly non-uniform velocity distribution, the numerical model reproduces both the symmetric and non-symmetric flow patterns as observed in the laboratory experiments. On one hand, for the geometries corresponding to an observed non-symmetric flow field, the numerical model converges towards a completely deviated flow field, the global pattern of which is consistent with laboratory observations. The result is essentially insensitive to the arbitrary amplitude of the small disturbances superimposed to the inflow boundary condition. On the other hand, for the geometries corresponding to an observed symmetric flow field, the slight disturbances introduced upstream are quickly damped and the computed steady flow field is found almost symmetric. Moreover, the numerical model accounts for wall roughness, which appears decisive for a proper reproduction of the third vortex, located in the upper part of the basin, in the case of non-symmetric flow fields. Consequently, for the tested configurations, the 2D simulations are found to succeed in predicting the influence of the length and width of the basin on the global flow pattern or, in other words, the influence of the expansion ratio $E R$ and of the aspect ratio $A R$ of the basin. Numerical and experimental results are compared by means of the first moment of the longitudinal velocity field about the centreline of the basin, which serves as a useful quantifier of "non-symmetry". Indeed, it remains equal to zero for all symmetric flow fields and increases with the dissymmetry of the flow pattern.

The influence of bottom roughness will be systematically analyzed in a subsequent work.

Finally, a linear analysis has been undertaken to appreciate the stability of transverse profiles of the longitudinal velocity in simulated symmetric flow fields. The system of $2 \mathrm{D}$ equations governing the flow has been simplified according to the rigid-lid approximation, which is known to be valid for Froude numbers as low as the present ones ( $F r \sim 0.1)$, leading to a modified Orr-Sommerfeld equation taking into consideration the Manning friction formula. A modified Rayleigh equation is obtained if turbulent diffusion is neglected.

An approximated solution in the form of a harmonic function has first been used to describe the relative importance of the damping effects for the two considered base flows (test no. 1 and test no. 2). The damping rate is shown to be weaker in the configuration which leads to a flow instability. Secondly, a finite difference scheme is used to discretize the modified Rayleigh equation, enabling to solve the corresponding eigenvalue problem. The deduced wave amplification factors lead to a clear distinction between an unstable and a more stable symmetric flow field for two different geometries, consistently with above-mentioned findings of physical and numerical modelling. This analysis focuses on the possible onset of an instability and cannot directly predict the length scales of the eddies observed experimentally. Therefore, the present appreciation should be confirmed by further investigations, possibly based on (weakly) non-linear stability analysis. 
More generally, the herein presented study recalls the practical importance in hydraulic engineering of complementing the computational design of hydraulic constructions by a careful verification of the stability of the expected flow fields.

Acknowledgements The authors gratefully acknowledge the Belgian National Fund for Scientific Research (F.R.S.-FNRS) and the University of Liege (ARD and Duesberg Foundation), which provided funding for the numerical and theoretical research carried out during post-doctoral studies at the LCH-EPFL.

\section{References}

1. Chen D, Jirka G (1997) Absolute and convective instabilities of plane turbulent wakes in a shallow water layer. J Fluid Mech 338:157-172

2. Ghidaoui MS, Kolyshkin AA (1999) Linear stability of lateral motions in compound open channels. J Hydraul Eng-ASCE 125(8):871-880

3. Chu VH, Wu J-H, Khayat RE (1991) Stability of transverse shear flows in shallow open channels. J Hydraul Eng-ASCE 117(10):1370-1388

4. Shapira M, Degani D, Weihs D (1990) Stability and existence of multiuple solutions for viscous flow in suddenly enlarged channels. Comput Fluids 18(3):239-258

5. Adamsson A, Stovin V, Bergdahl L (2003) Bed shear stress boundary condition for storage tank sedimentation. J Environ Eng-ASCE 129(7):651-658

6. Stovin VR, Saul AJ (1996) Efficiency prediction for storage chambers using computational fluid dynamics. Water Sci Tech 33(9):163-170

7. Kantoush S (2007) Symmetric or asymmetric flow patterns in shallow rectangular basins with sediment transport. In: Proc. 32nd IAHR biennial congress, Venice, Italy

8. Kantoush SA, Bollaert EFR, Boillat J-L, Schleiss AJ, Uijttewaal WSJ (2006) Sedimentation processes in shallow reservoirs with different geometries. In: Proc. int. conf. on fluvial hydraulics, Lisboa, Portugal

9. Kantoush S, Bollaert E, de Cesare G, Boillat J-L, Schleiss A (2006) Flow field investigation in a rectangular shallow reservoir using UVP, LSPIV and numerical model. In: Proc. 5th int. symp. on ultrasonic Doppler methods for fluid mechanics and fluid engineering, ETH Zurich, Switzerland

10. Kolyshkin AA, Ghidaoui MS (2003) Stability analysis of shallow wake flows. J Fluid Mech 494:355-377

11. Chen D, Jirka GH (1995) Experimental study of plane turbulent wakes in a shallow water layer. Fluid Dyn Res 16:11-41

12. Mizushima J, Okamoto H, Yamaguchi H (1996) Stability of flow in a channel with a suddenly expanded part. Phys Fluids 8(11):2933-2942

13. Mizushima J, Shiotani Y (2001) Transitions and instabilities of flow in a symmetric channel with a suddenly expanded and contracted part. J Fluid Mech 434:355-369

14. Battaglia F, Tavener SJ, Kulkarni AK, Merkle CL (1997) Bifurcation of low reynolds number flows in symmetric channels. AIAA J 35:99-105

15. Kantoush SA, Bollaert E, Boillat JL, Schleiss A (2005) Suspended load transport in shallow reservoirs. In: Proc. 31st IAHR biennial congress. Korea Water Ressources Association, Seoul, South Korea

16. Dewals BJ (2006) Une approche unifiée pour la modélisation d'écoulements à surface libre, de leur effet érosif sur une structure et de leur interaction avec divers constituants. PhD Thesis, University of Liege, Liège, p 636

17. Erpicum S (2006) Optimisation objective de paramètres en écoulements à surface libre sur maillage multibloc. PhD Thesis, University of Liege, p 364

18. Drazin PG, Reid WH (1981) Hydrodynamic stability. In: Batchelor GK, Miles JW (eds) Cambridge monographs on mechanics and applied mathematics. Cambridge University Press

19. Betchov R, Criminale WO (1967) Stability of parallel flows. In: Frenkiel FN, Temple G (eds) Applied mathematics and mechanics. Academic Press

20. Chiang TP, Sheu TWH, Wang SK (2000) Side wall effects on the structure of laminar flow over a planesymmetric sudden expansion. Comput Fluids 29:467-492

21. Chaudhry MH (1993) Open-channel flow. Prentice-Hall

22. ASCE Task Committee on Turbulence Models in Hydraulic Computations (1988) Turbulence modeling of surface water flow and transport: part I. J Hydraul Eng-ASCE 114(9):970-991

23. Rodi W (1984) Turbulence models and their application in hydraulics - a state of the art review. IAHR, Delft, $104 \mathrm{pp}$ 
24. Fischer HB, List EJ, Koh RCY, Imberger J, Brooks NH (1979) Mixing in inland and coastal waters. Academic Press (Subtitle 1.4.7), pp 21-22

25. Dewals BJ, Erpicum S, Archambeau P, Detrembleur S, Pirotton M (2006) Depth-integrated flow modelling taking into account bottom curvature. J Hydraul Res 44(6):787-795

26. Dewals BJ, Erpicum S, Archambeau P, Detrembleur S, Pirotton M (2006) Numerical tools for dam break risk assessment: validation and application to a large complex of dams. In: Hewlett H (ed) Improvements in reservoir construction, operation and maintenance. Thomas Telford, London, pp 272-282

27. Riahi DN (2000) Flow instability. WIT Press, 224 pp

28. Shivamoggi BK (1998) Theoretical fluid dynamics. Wiley, New York, p 555

29. Prooijen BCvan, Uijttewaal WS (2002) A linear approach for the evolution of coherent structures in shallow mixing layers. Phys Fluids 14(2):4105-4114

30. Orszag SA (1971) Accurate solution of the Orr-Sommerfeld stability equation. J Fluid Mech 50(4):689703 